\title{
Supporting Information: A Combined Infrared Ion Spectroscopy and Computational Chemistry Study of Hydroxyproline Isomers
}

Baku Acharya, ${ }^{\mathrm{a} \dagger}$ W. K. D. N. Kaushalya ${ }^{\mathrm{a} \dagger}{ }^{\dagger}$ Jonathan Martens, ${ }^{\mathrm{b}}$ Giel Berden, ${ }^{\mathrm{b}}$ Jos Oomens, ${ }^{\mathrm{b}}$ and Amanda L. Patrick ${ }^{\mathrm{a} *}$

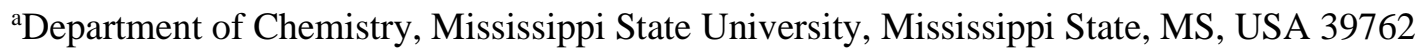

${ }^{\mathrm{b}}$ Radboud University, Institute for Molecules and Materials, FELIX Laboratory, Toernooiveld 7, 6525ED Nijmegen, The Netherlands

$\dagger$ Authors contributed equally.

KEYWORDS: IRMPD spectroscopy, hydroxyproline, lithium, zwitterion

*Corresponding Author: Dr. Amanda Patrick, apatrick@ chemistry.msstate.edu

Note: The authors declare no conflicts of interest. 


\section{Table of Contents:}

Figure S1: Laser induced dissociation (LID) mass spectra of (A) protonated cis-3-hydroxyproline (left) and protonated cis-4-hydroxyproline (right) and (B) LID mass spectra of lithiated cis-3hydroxyproline (top panel), lithiated cis-4-hydroxyproline (middle panel), and lithiated trans-4hydroxyproline (bottom panel). Extensive dissociation is seen when a higher laser power is used at an on-resonance wavenumber ("Laser ON, High Power"; left column), while less extensive dissociation is observed when lower powers were used at on-resonance wavenumbers ("Laser ON, Low Power"; middle column). No dissociation is seen at off-resonance wavenumbers ("Laser OFF"; third column).

Figure S2: Experimental IRMPD spectrum of lithiated cis-3-hydroxyproline (A) and theoretical infrared spectra of the most energetically stable (charge solvation) conformer (B), of two higher energy charge solvation conformers $(\mathrm{C}, \mathrm{D})$, and of the three most energetically stable salt bridge conformers (E-G).

Figure S3: Structures of three most stable conformers of charge solvation form (left) and saltbridge form (right) of lithiated cis-3-hydroxyproline.

Figure S4: Experimental IRMPD spectrum of lithiated cis-4-hydroxyproline (A) and theoretical infrared spectra of the most energetically stable (charge solvation) conformer (B), of two higher energy charge solvation conformers $(\mathrm{C}, \mathrm{D})$, and of the three most energetically stable salt bridge conformers (E-G).

Figure S5: Structures of three most stable conformers of charge solvation form (left) and saltbridge form (right) of lithiated cis-4-hydroxyproline.

Figure S6: Experimental IRMPD spectrum of lithiated trans-4-hydroxyproline (A) and theoretical infrared spectra of the most energetically stable (salt bridge) conformer (B), of two higher energy salt bridge conformers $(\mathrm{C}, \mathrm{D})$, and of the three most energetically charge solvation conformers (E-G).

Figure S7: Structures of three most stable conformers of salt bridge form (left) and charge solvation form (right) of lithiated trans-4-hydroxyproline

Figure S8: Theoretical infrared spectra of the most stable charge solvation (A) and salt bridge (B) conformers of lithiated trans-4-hydroxyproline and charge solvation $(\mathrm{C})$ and salt bridge (D) conformers of lithiated trans-3-hydroxyproline

Figure S9: IRMPD spectrum of lithiated cis-3-hydroxyproline (A) and the theoretical infrared spectra of the most stable charge solvation (B) and salt bridge $(\mathrm{C})$ conformers at M062X level with the basis set 6-31++G(d, p).

Figure S10: IRMPD spectrum of lithiated cis-4-hydroxyproline (A) and the theoretical infrared spectra of the most stable charge solvation $(B)$ and salt bridge $(\mathrm{C})$ conformers at M062X level with the basis set 6-31++G(d, p). 
Figure S11: IRMPD spectrum of lithiated trans-4-hydroxyproline (A) and the theoretical infrared spectra of the most stable salt bridge (B) and charge solvation (C) conformers at M062X level with the basis set 6-31++G(d, p).

Section S1. Coordinates of the optimized structures of three most stable conformers of chargesolvated and salt-bridge of lithiated cis-3-hydroxyproline at B3LYP level with the basis set 6$31++\mathrm{G}(\mathrm{d}, \mathrm{p})$

Section S2. Coordinates of the optimized structures of three most stable conformers of chargesolvated and salt-bridge of lithiated cis-4-hydroxyproline at B3LYP level with the basis set 6$31++\mathrm{G}(\mathrm{d}, \mathrm{p})$

Section S3. Coordinates of the optimized structures of three most stable conformers of chargesolvated and salt-bridge of lithiated trans-4-hydroxyproline at B3LYP level with the basis set 6-31++G(d, p)

Section S4. Coordinates of the optimized structure of the lowest energy lithiated cis-3hydroxyproline structures for the charge solvated (C3CS01) and salt-bridge (C3SB01) conformations at M062X level with the basis set 6-31++G(d, p)

Section S5. Coordinates of the optimized structure of the lowest energy lithiated cis-4hydroxyproline structures for the charge solvated (C4CS01) and salt-bridge (C4SB01) conformations at M062X level with the basis set 6-31++G(d, p)

Section S6. Coordinates of the optimized structure of the lowest energy lithiated trans-4hydroxyproline structures for the charge solvated (T4CS01) and salt-bridge (T4SB01) conformations at M062X level with the basis set 6-31++G(d,p)

Section S7. Coordinates of the optimized structure of the lowest energy protonated cis-3hydroxyproline structure at B3LYP level with the basis set $6-31++G(d, p)$

Section S8. Coordinates of the optimized structure of the lowest energy protonated cis-4hydroxyproline structure at B3LYP level with the basis set 6-31++G(d, p)

Table S1: Experimental and theoretical infrared peaks $\left(\mathrm{cm}^{-1}\right)$ along with the vibrational modes for each of the three lithiated hydroxyproline isomers 
(A)

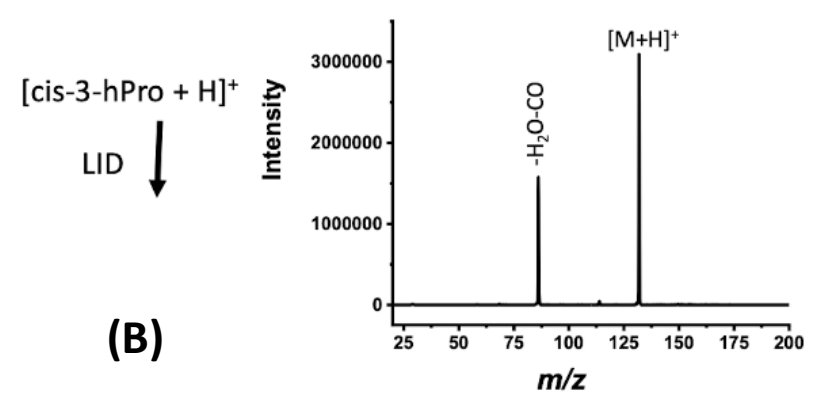

\section{Laser ON}

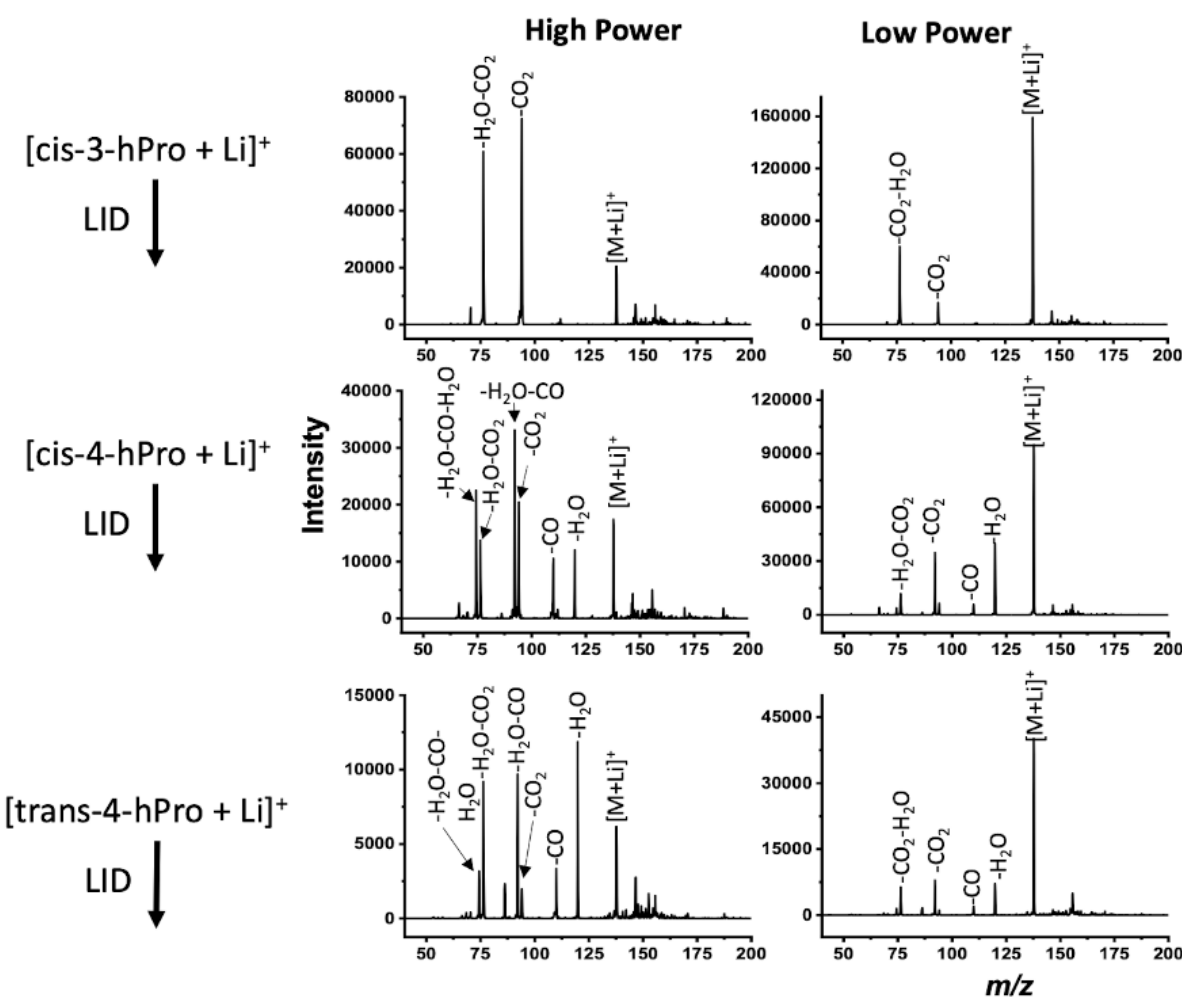

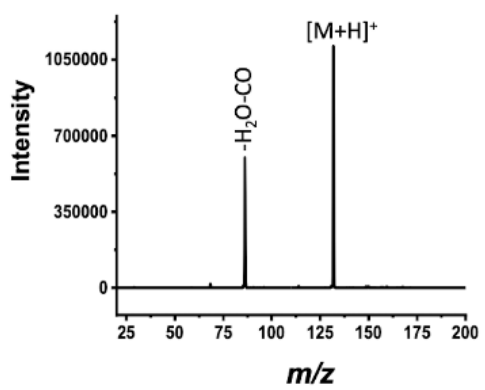

Laser OFF
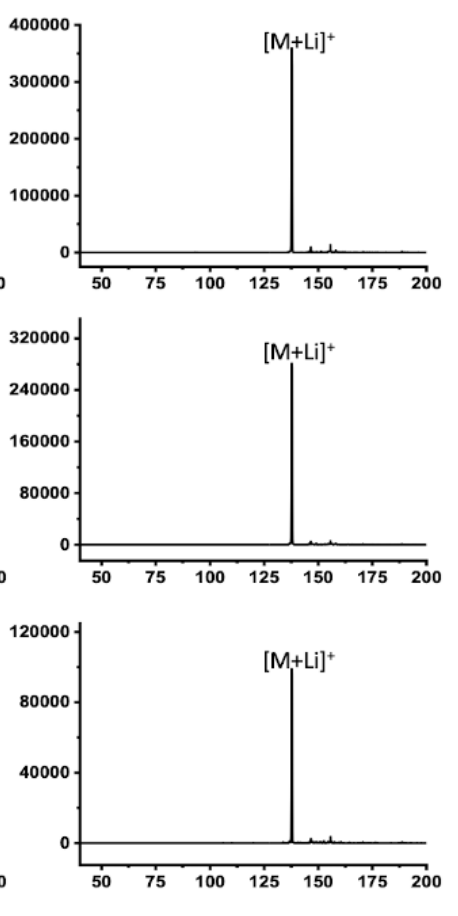

Figure S1: Laser induced dissociation (LID) mass spectra of (A) protonated cis-3-hydroxyproline (left) and protonated cis-4-hydroxyproline (right) and (B) LID mass spectra of lithiated cis-3-hydroxyproline (top panel), lithiated cis-4-hydroxyproline (middle panel), and lithiated trans-4-hydroxyproline (bottom panel). For Part B: Extensive dissociation is seen when a higher laser power is used at an on-resonance wavenumber ("Laser ON, High Power"; left column), while less extensive dissociation is observed when lower powers were used at on-resonance wavenumbers ("Laser ON, Low Power"; middle column). No dissociation is seen at off-resonance wavenumbers ("Laser OFF"; third column). 


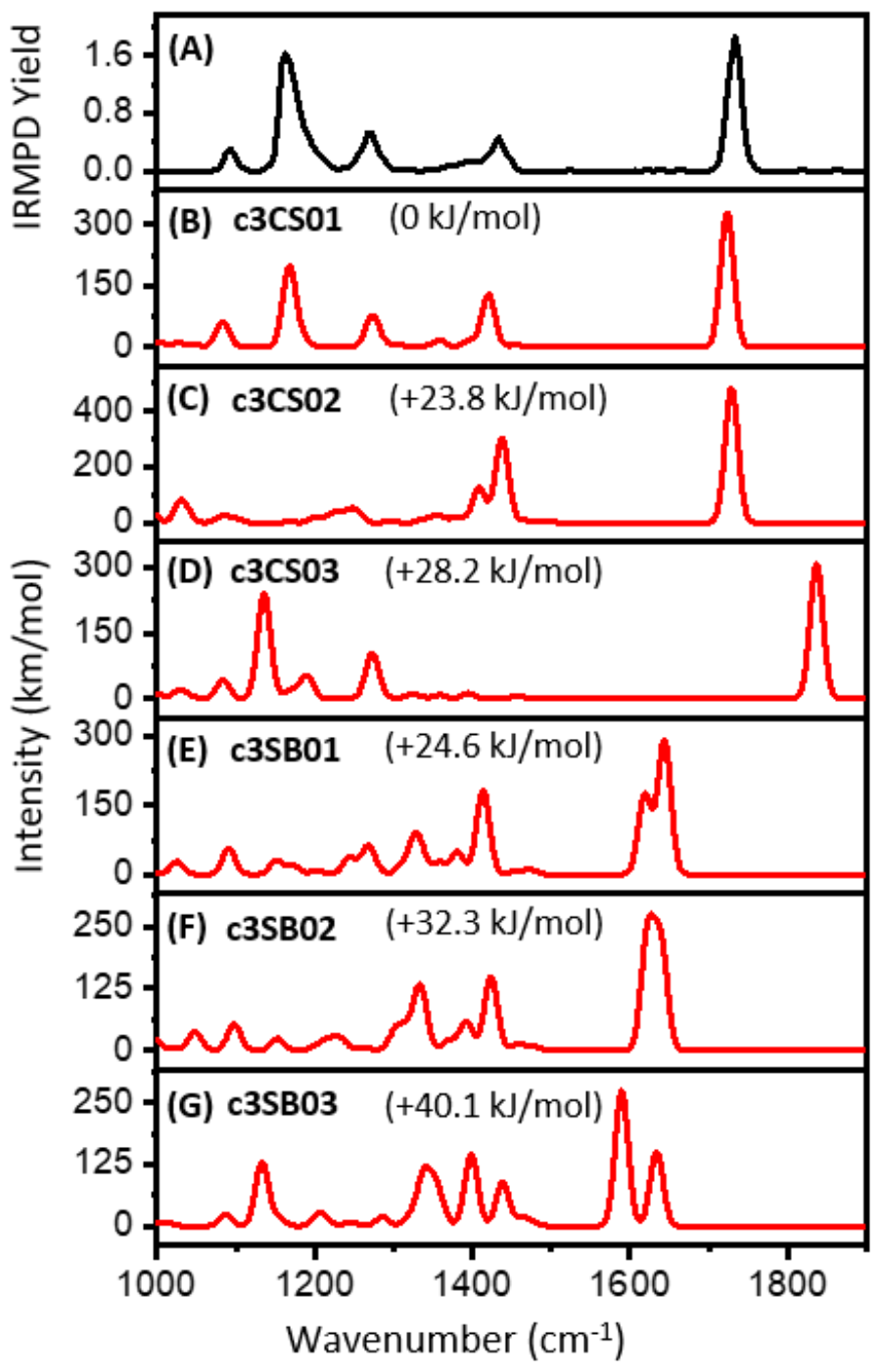

Figure S2: Experimental IRMPD spectrum of lithiated cis-3-hydroxyproline (A) and theoretical infrared spectra of the most energetically stable (charge solvation) conformer $(B)$, of two higher energy charge solvation conformers $(C, D)$, and of the three most energetically stable salt bridge conformers $(E-G)$. 


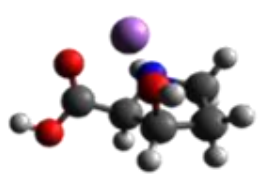

c3CS01

$(0 \mathrm{~kJ} / \mathrm{mol})$

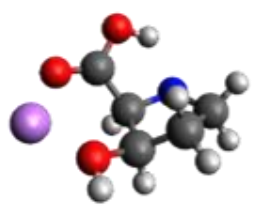

c3CS02

(+23.8 kJ/mol)

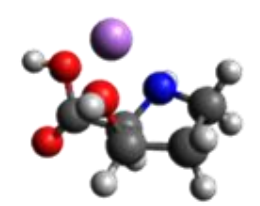

c3CS03

(+28.2 kJ/mol)

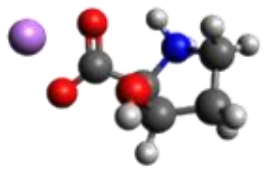

c3SB01

$(+24.6 \mathrm{~kJ} / \mathrm{mol})$

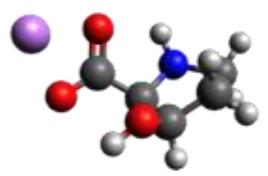

c3SB02

(+32.3 kJ/mol)

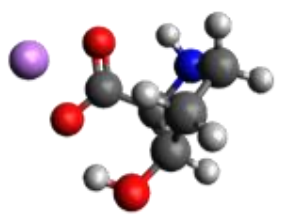

c3SB03

(+40.1 kJ/mol)

Figure S3: Structures of three most stable conformers of charge solvation form (left) and saltbridge form (right) of lithiated cis-3-hydroxyproline 


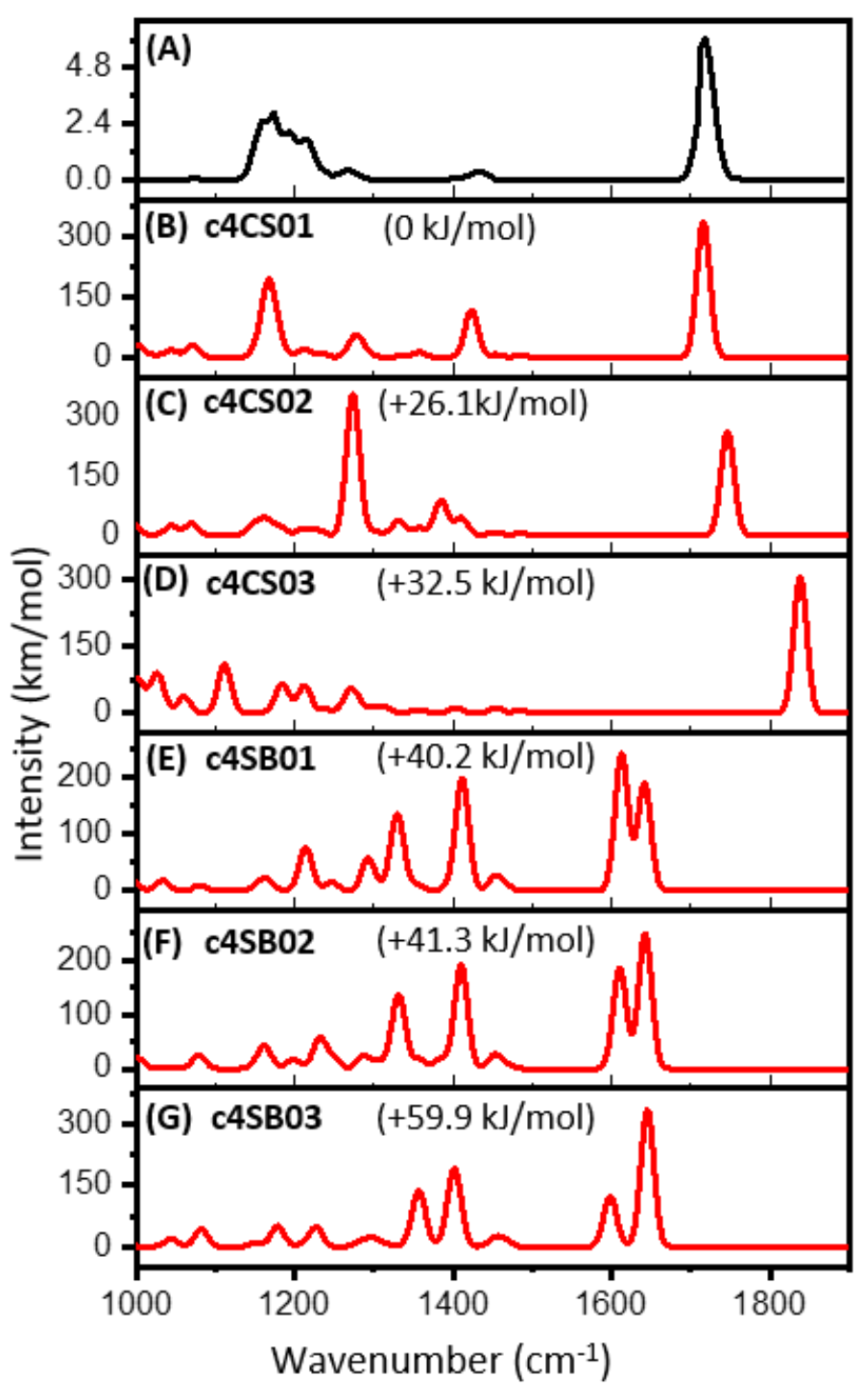

Figure S4: Experimental IRMPD spectrum of lithiated cis-4-hydroxyproline (A) and theoretical infrared spectra of the most energetically stable (charge solvation) conformer (B), of two higher energy charge solvation conformers $(C, D)$, and of the three most energetically stable salt bridge conformers $(E-G)$. 


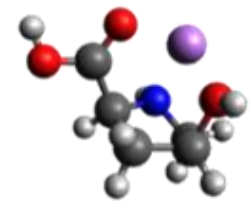

c4CS01

$(0 \mathrm{~kJ} / \mathrm{mol})$

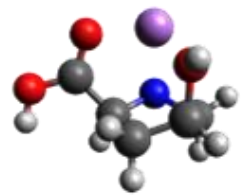

c4CS02

(+26.1 $\mathrm{kJ} / \mathrm{mol})$

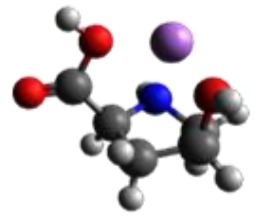

c4CS03

(+32.5 kJ/mol)

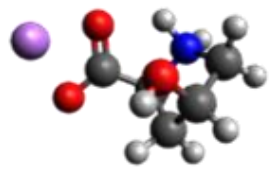

c4SB01

(+40.2 kJ/mol)

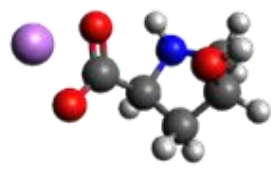

c4SB02

$(+41.3 \mathrm{~kJ} / \mathrm{mol})$

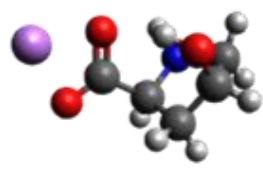

c4SB03

(+59.9 kJ/mol)

Figure S5: Structures of three most stable conformers of charge solvation form (left) and saltbridge form (right) of lithiated cis-4-hydroxyproline 


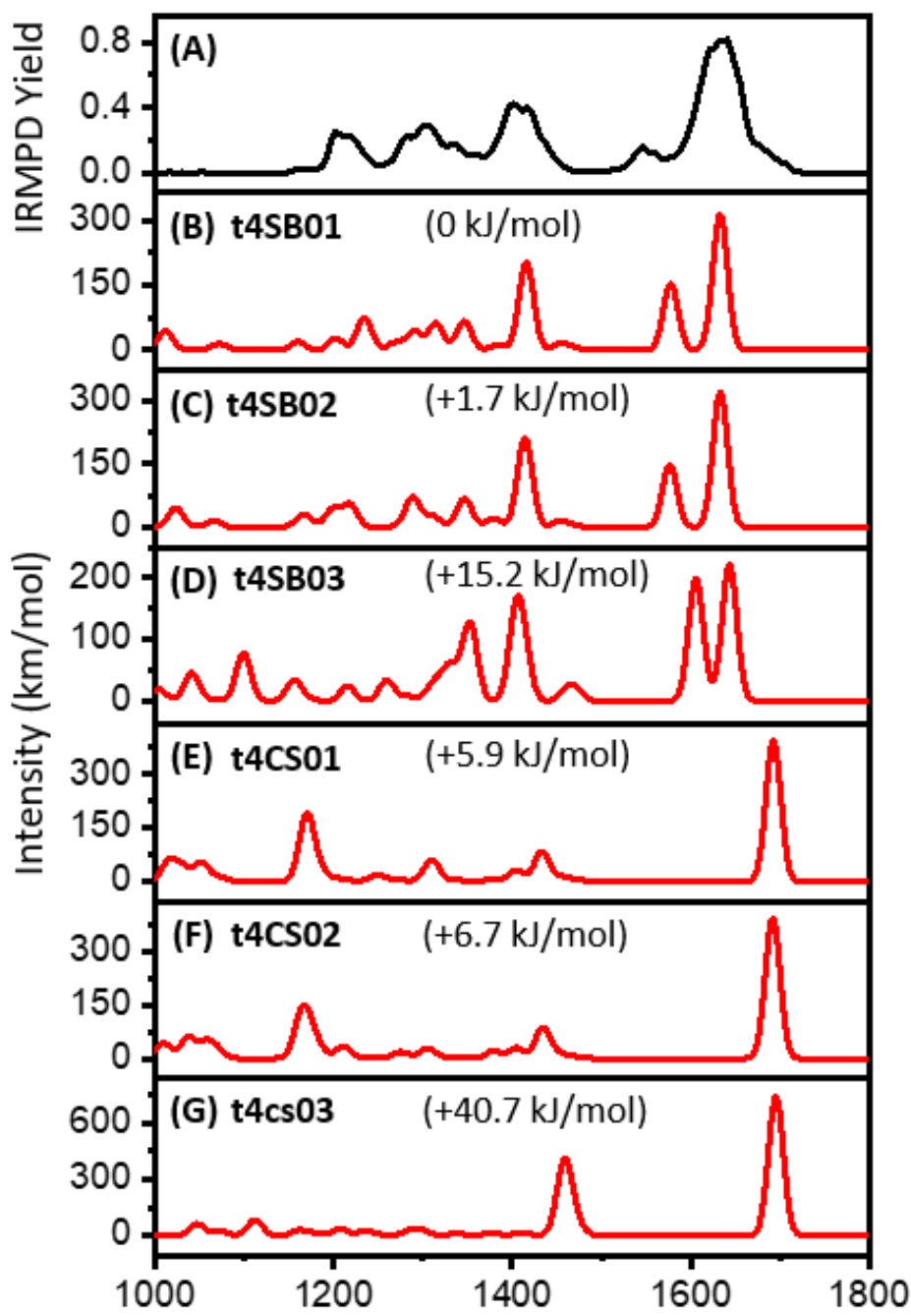

Figure S6: Experimental IRMPD spectrum of lithiated trans-4-hydroxyproline (A) and theoretical infrared spectra of the most energetically stable (salt bridge) conformer (B), of two higher energy salt bridge conformers $(C, D)$, and of the three most energetically charge solvation conformers $(E-G)$. 


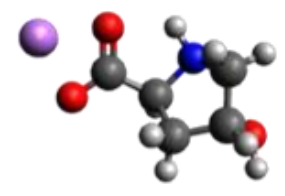

t4SB01

(0 kJ/mol)

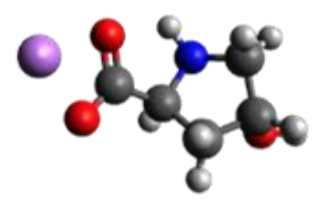

t4SB02

$(+1.7 \mathrm{~kJ} / \mathrm{mol})$

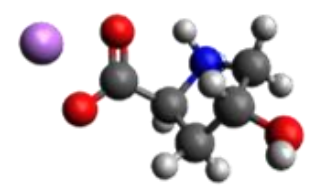

t4SB03

$(+15.2 \mathrm{~kJ} / \mathrm{mol})$

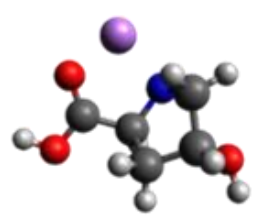

t4CS01

(+5.9 kJ/mol)

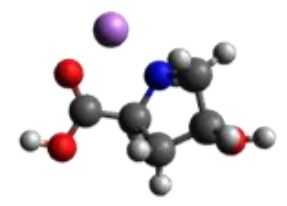

t4CS02

$(+6.7 \mathrm{~kJ} / \mathrm{mol})$

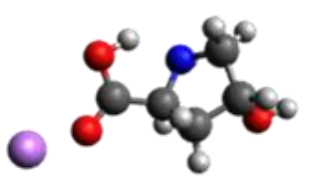

t4CS03

$(+40.7 \mathrm{~kJ} / \mathrm{mol})$

Figure S7: Structures of three most stable conformers of salt bridge form (left) and charge solvation form (right) of lithiated trans-4-hydroxyproline 


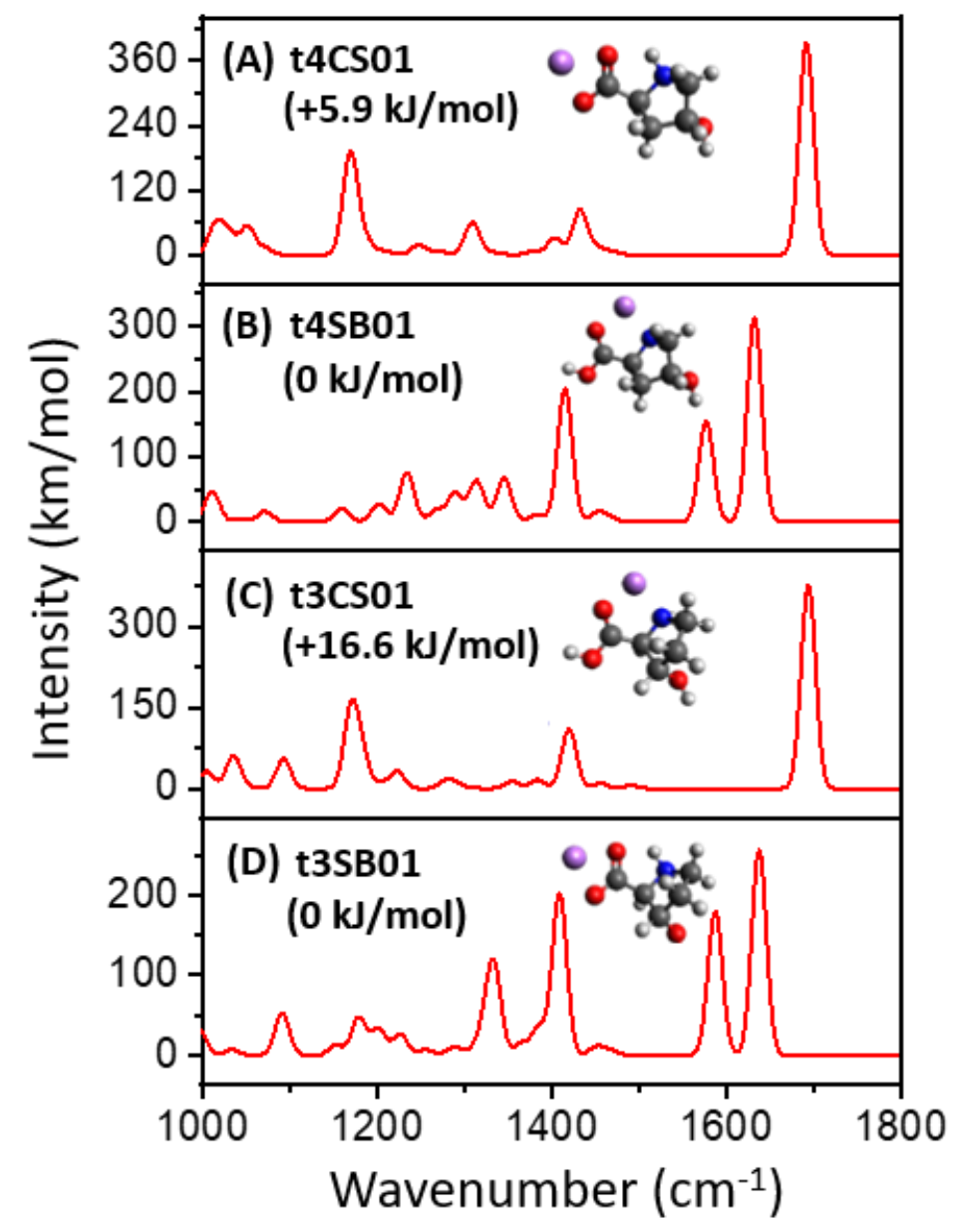

Figure S8: Theoretical infrared spectra of the most stable charge solvation (A) and salt bridge $(B)$ conformers of lithiated trans-4-hydroxyproline and charge solvation $(C)$ and salt bridge $(D)$ conformers of lithiated trans-3-hydroxyproline 


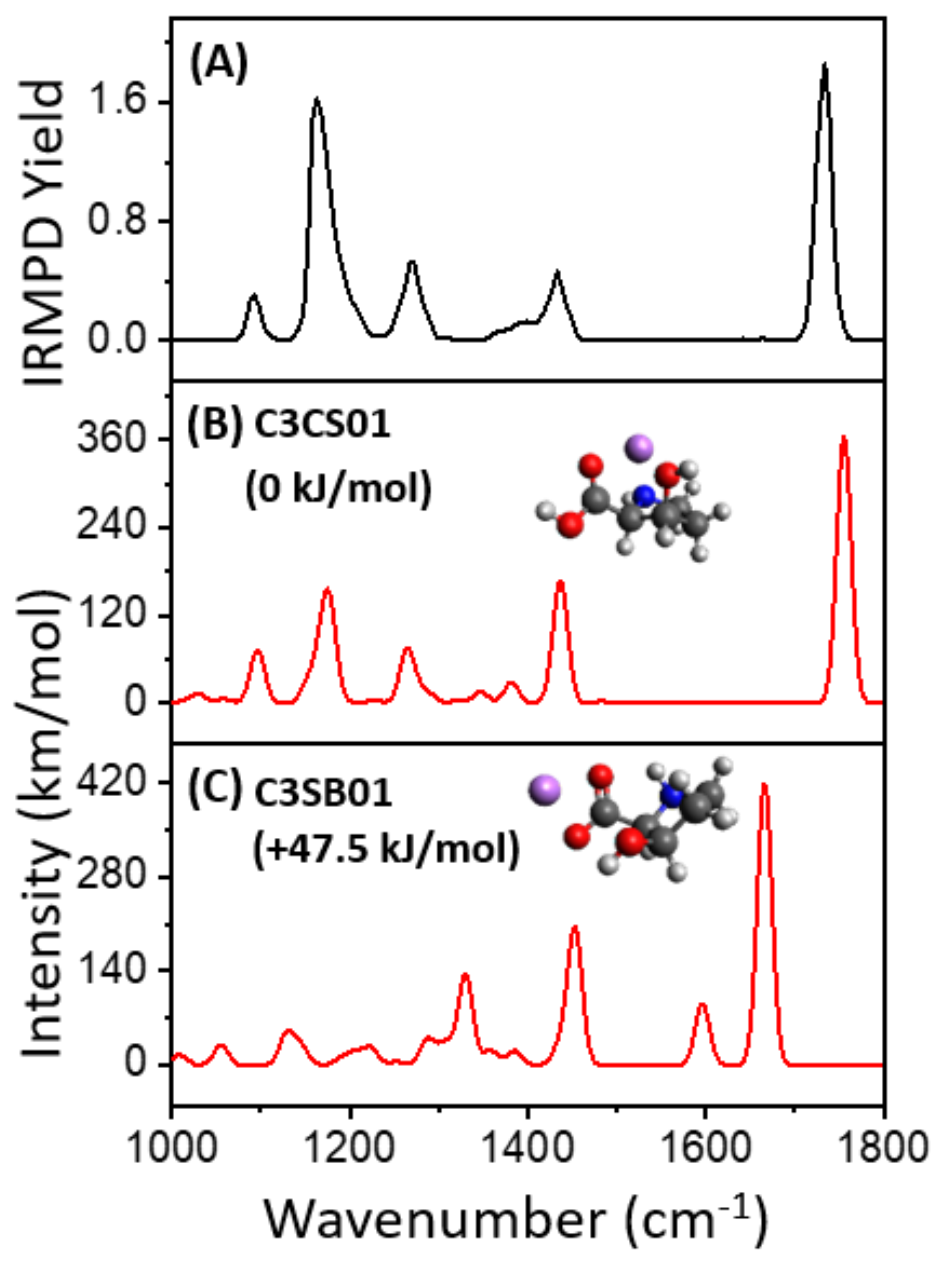

Figure S9: IRMPD spectrum of lithiated cis-3-hydroxyproline (A) and the theoretical infrared spectra of the most stable charge solvation $(B)$ and salt bridge $(C)$ conformers at M062X level with the basis set $6-31++G(d, p)$. 


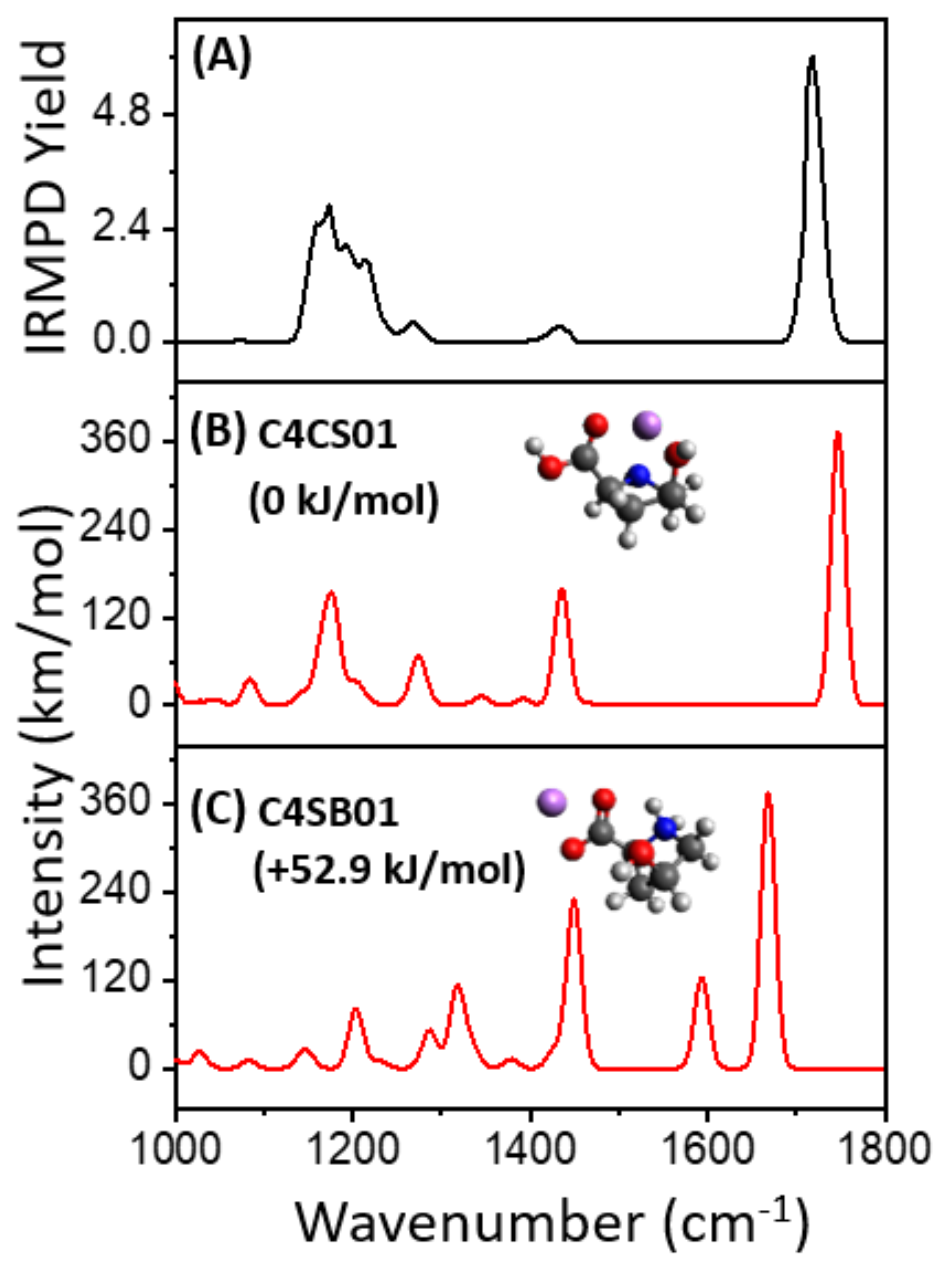

Figure S10: IRMPD spectrum of lithiated cis-4-hydroxyproline $(A)$ and the theoretical infrared spectra of the most stable charge solvation $(B)$ and salt bridge $(C)$ conformers at M062X level with the basis set $6-31++G(d, p)$. 


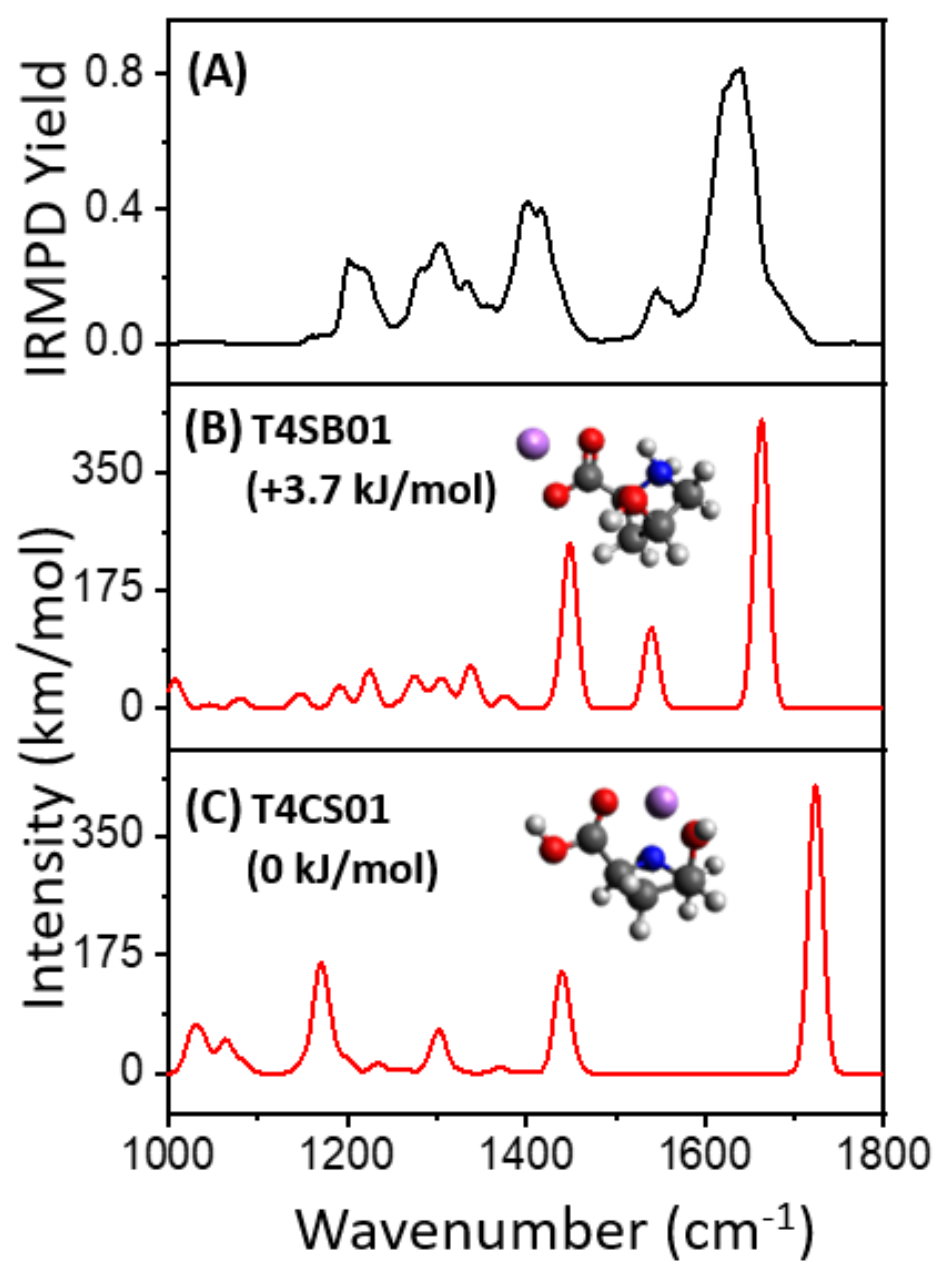

Figure S11: IRMPD spectrum of lithiated trans-4-hydroxyproline (A) and the theoretical infrared spectra of the most stable salt bridge $(B)$ and charge solvation $(C)$ conformers at M062X level with the basis set 6-31++G(d,p). 
Section S1. Coordinates of the optimized structures of three most stable conformers of charge-solvated and salt-bridge of lithiated cis-3-hydroxyproline at B3LYP level with the basis set 6-31++G(d, p)

\section{c3CS01}

$\begin{array}{lrrr}1 & 1 & & \\ \mathrm{C} & 0.143729 & -0.428311 & -0.548830 \\ \mathrm{C} & -0.687261 & 0.875663 & -0.614691 \\ \mathrm{C} & -2.120388 & 0.331771 & -0.665293 \\ \mathrm{C} & -2.061861 & -0.993012 & 0.141612 \\ \mathrm{~N} & -0.613925 & -1.149029 & 0.498369 \\ \mathrm{C} & 1.578238 & -0.206022 & -0.091181 \\ \mathrm{H} & -0.410407 & 1.526551 & -1.446789 \\ \mathrm{H} & -2.406933 & 0.148323 & -1.704916 \\ \mathrm{O} & 2.442354 & -0.108622 & -1.087287 \\ \mathrm{O} & 1.877611 & -0.104607 & 1.094078 \\ \mathrm{H} & 0.139280 & -0.912657 & -1.533469 \\ \mathrm{O} & -0.434026 & 1.547004 & 0.648514 \\ \mathrm{H} & -0.349522 & -2.130842 & 0.549816 \\ \mathrm{H} & -2.412653 & -1.835149 & -0.463135 \\ \mathrm{H} & -2.659744 & -0.964122 & 1.055221 \\ \mathrm{H} & -2.831307 & 1.052300 & -0.253137 \\ \mathrm{H} & -0.801485 & 2.440845 & 0.643762 \\ \mathrm{Li} & 0.163711 & 0.166872 & 1.943450 \\ \mathrm{H} & 3.336852 & 0.066599 & -0.738423\end{array}$

\section{c3CS02}

$\begin{array}{lrrr}1 & 1 & & \\ \mathrm{C} & -0.023748 & -0.272194 & -0.793151 \\ \mathrm{C} & -0.524143 & 1.131426 & -0.356542 \\ \mathrm{C} & -1.533558 & 0.816452 & 0.750046 \\ \mathrm{C} & -2.244391 & -0.421495 & 0.188893 \\ \mathrm{~N} & -1.162347 & -1.165020 & -0.526778 \\ \mathrm{C} & 1.196959 & -0.793330 & -0.000902 \\ \mathrm{H} & -1.025587 & 1.589032 & -1.216087 \\ \mathrm{H} & -2.226446 & 1.638790 & 0.946118 \\ \mathrm{O} & 2.276433 & -0.197854 & 0.026363 \\ \mathrm{O} & 1.002631 & -1.925288 & 0.613916 \\ \mathrm{H} & 0.274179 & -0.275957 & -1.847668 \\ \mathrm{O} & 0.555439 & 2.016786 & 0.042583 \\ \mathrm{H} & -1.499782 & -1.636862 & -1.359632 \\ \mathrm{H} & -3.041805 & -0.130662 & -0.504103 \\ \mathrm{H} & -2.682635 & -1.047053 & 0.969251 \\ \mathrm{H} & -1.002962 & 0.583571 & 1.680719 \\ \mathrm{H} & 0.173583 & 2.881131 & 0.253589 \\ \mathrm{Li} & 2.407812 & 1.595672 & 0.049159 \\ \mathrm{H} & 0.041707 & -2.148179 & 0.424826\end{array}$




\section{c3CS03}

$\begin{array}{lccc}1 & 1 & & \\ \mathrm{C} & 0.122497 & -0.308174 & -0.659584 \\ \mathrm{C} & -0.702366 & 0.977683 & -0.439590 \\ \mathrm{C} & -2.140445 & 0.451211 & -0.519159 \\ \mathrm{C} & -2.047450 & -1.010900 & -0.002507 \\ \mathrm{~N} & -0.585412 & -1.218023 & 0.272860 \\ \mathrm{C} & 1.604634 & -0.139743 & -0.352641 \\ \mathrm{H} & -0.457458 & 1.776150 & -1.142272 \\ \mathrm{H} & -2.486811 & 0.481449 & -1.556059 \\ \mathrm{O} & 2.441735 & 0.196356 & -1.140335 \\ \mathrm{O} & 1.886764 & -0.391078 & 0.978745 \\ \mathrm{H} & 0.051143 & -0.603722 & -1.713937 \\ \mathrm{O} & -0.383614 & 1.398535 & 0.919542 \\ \mathrm{H} & -0.319795 & -2.190037 & 0.126238 \\ \mathrm{H} & -2.406882 & -1.716184 & -0.758305 \\ \mathrm{H} & -2.618898 & -1.180193 & 0.912795 \\ \mathrm{H} & -2.821364 & 1.069823 & 0.070593 \\ \mathrm{H} & -0.708837 & 2.292658 & 1.089629 \\ \mathrm{Li} & 0.148740 & -0.201408 & 1.904659 \\ \mathrm{H} & 2.840258 & -0.250522 & 1.124589\end{array}$

\section{c3SB01}

$\begin{array}{lrrr}1 & 1 & & \\ \mathrm{C} & 0.025877 & -0.150303 & -0.755496 \\ \mathrm{C} & -0.847168 & 1.043666 & -0.316613 \\ \mathrm{C} & -2.261197 & 0.434910 & -0.290646 \\ \mathrm{C} & -2.039317 & -0.924330 & 0.377947 \\ \mathrm{~N} & -0.677341 & -1.364315 & -0.156784 \\ \mathrm{C} & 1.463074 & -0.129904 & -0.221352 \\ \mathrm{H} & -0.742398 & 1.868805 & -1.026708 \\ \mathrm{H} & -2.649256 & 0.317678 & -1.308988 \\ \mathrm{O} & 2.261856 & 0.729891 & -0.656638 \\ \mathrm{O} & 1.766129 & -1.008885 & 0.651238 \\ \mathrm{H} & 0.030029 & -0.242189 & -1.843364 \\ \mathrm{O} & -0.427804 & 1.413453 & 0.990279 \\ \mathrm{H} & -0.767707 & -2.115276 & -0.843388 \\ \mathrm{H} & -2.781212 & -1.685657 & 0.137308 \\ \mathrm{H} & -1.942994 & -0.830961 & 1.459590 \\ \mathrm{H} & -2.963741 & 1.053184 & 0.272583 \\ \mathrm{H} & -0.656529 & 2.335078 & 1.169041 \\ \mathrm{Li} & 3.462587 & -0.057520 & 0.620162 \\ \mathrm{H} & -0.021625 & -1.697797 & 0.578858\end{array}$




$\begin{array}{lrrr}\text { c3SB02 } & & & \\ 1 & 1 & & \\ \mathrm{C} & -0.029521 & -0.213041 & -0.792892 \\ \mathrm{C} & -0.823561 & 1.076676 & -0.341194 \\ \mathrm{C} & -1.960356 & 0.546250 & 0.552241 \\ \mathrm{C} & -2.231553 & -0.874349 & 0.075583 \\ \mathrm{~N} & -0.846198 & -1.382564 & -0.276729 \\ \mathrm{C} & -1.360935 & -0.304762 & -0.160498 \\ \mathrm{H} & -1.220672 & 1.564640 & -1.239214 \\ \mathrm{H} & -2.853143 & 1.171820 & 0.498646 \\ \mathrm{O} & 2.262480 & 0.467275 & -0.566661 \\ \mathrm{O} & 1.522432 & -1.156581 & 0.773185 \\ \mathrm{H} & 0.049385 & -0.281038 & -1.879102 \\ \mathrm{O} & -0.056487 & 1.972955 & 0.421854 \\ \mathrm{H} & -0.872227 & -2.162112 & -0.938120 \\ \mathrm{H} & -2.828935 & -0.905743 & -0.838763 \\ \mathrm{H} & -2.677725 & -1.535036 & 0.820174 \\ \mathrm{H} & -1.606123 & 0.551901 & 1.588587 \\ \mathrm{H} & 0.605993 & 2.409734 & -0.133768 \\ \mathrm{Li} & 3.303837 & -0.360287 & 0.826628 \\ \mathrm{H} & -0.307739 & -1.709203 & 0.552317\end{array}$

\section{c3SB03}

$\begin{array}{lrrr}1 & 1 & & \\ \mathrm{C} & -0.046339 & -0.090924 & -0.863229 \\ \mathrm{C} & -0.992915 & 1.012938 & -0.300195 \\ \mathrm{C} & -1.625102 & 0.309233 & 0.919693 \\ \mathrm{C} & -1.934759 & -1.091425 & 0.398229 \\ \mathrm{~N} & -0.791774 & -1.383910 & -0.597202 \\ \mathrm{C} & 1.298301 & -0.210277 & -0.129512 \\ \mathrm{H} & -1.778564 & 1.187082 & -1.047536 \\ \mathrm{H} & -2.527107 & 0.820793 & 1.261005 \\ \mathrm{O} & 2.011616 & 0.819396 & 0.012458 \\ \mathrm{O} & 1.639767 & -1.354891 & 0.304023 \\ \mathrm{H} & 0.142258 & 0.021912 & -1.933549 \\ \mathrm{O} & -0.392069 & 2.246486 & -0.061770 \\ \mathrm{H} & -1.142606 & -1.816120 & -1.453691 \\ \mathrm{H} & -2.866099 & -1.125924 & -0.169544 \\ \mathrm{H} & -1.942712 & -1.880363 & 1.150148 \\ \mathrm{H} & -0.915714 & 0.281672 & 1.754279 \\ \mathrm{H} & 0.551455 & 2.132292 & 0.151117 \\ \mathrm{Li} & 3.275610 & -0.395414 & 0.821619 \\ \mathrm{H} & -0.074950 & -2.012925 & -0.184270\end{array}$


Section S2. Coordinates of the optimized structures of three most stable conformers of charge-solvated and salt-bridge of lithiated cis-4-hydroxyproline at B3LYP level with the basis set 6-31++G(d, p)

\section{c4CS01}

$\begin{array}{lrrr}1 & 1 & & \\ \mathrm{C} & -0.432833 & 0.864678 & 0.190185 \\ \mathrm{C} & 0.437852 & 0.827515 & -1.117428 \\ \mathrm{C} & 1.735134 & 0.135084 & -0.666954 \\ \mathrm{C} & 1.871331 & 0.611948 & 0.771553 \\ \mathrm{~N} & 0.486632 & 0.365959 & 1.249202 \\ \mathrm{C} & -1.622342 & -0.080158 & 0.078267 \\ \mathrm{H} & 0.653656 & 1.847148 & -1.446609 \\ \mathrm{H} & 2.599728 & 0.354743 & -1.296073 \\ \mathrm{O} & -2.712112 & 0.499079 & -0.396519 \\ \mathrm{O} & -1.556019 & -1.274151 & 0.361726 \\ \mathrm{H} & -0.800900 & 1.877651 & 0.379296 \\ \mathrm{H} & -0.049443 & 0.310136 & -1.947528 \\ \mathrm{O} & 1.538298 & -1.307139 & -0.575245 \\ \mathrm{H} & 1.731493 & -1.737890 & -1.418789 \\ \mathrm{H} & 0.298102 & 0.798657 & 2.150113 \\ \mathrm{H} & 2.159893 & 1.670781 & 0.798879 \\ \mathrm{H} & 2.592672 & 0.030636 & 1.350321 \\ \mathrm{Li} & 0.246616 & -1.684274 & 0.841346 \\ \mathrm{H} & -3.427657 & -0.157472 & -0.491488\end{array}$

\section{c4CS02}

$\begin{array}{lrrr}1 & 1 & & \\ \mathrm{C} & 0.455920 & -0.846558 & 0.196259 \\ \mathrm{C} & -0.418386 & -0.830340 & -1.110961 \\ \mathrm{C} & -1.733015 & -0.168433 & -0.658857 \\ \mathrm{C} & -1.849834 & -0.638735 & 0.783786 \\ \mathrm{~N} & -0.469252 & -0.357297 & 1.250395 \\ \mathrm{C} & 1.636344 & 0.125002 & 0.074458 \\ \mathrm{H} & -0.613926 & -1.853596 & -1.442291 \\ \mathrm{H} & -2.592615 & -0.420511 & -1.282755 \\ \mathrm{O} & 2.796401 & -0.330077 & -0.379934 \\ \mathrm{O} & 1.510405 & 1.313924 & 0.332057 \\ \mathrm{H} & 0.820954 & -1.861380 & 0.400511 \\ \mathrm{H} & 0.053137 & -0.296372 & -1.940018 \\ \mathrm{O} & -1.577912 & 1.276767 & -0.581465 \\ \mathrm{H} & -1.781750 & 1.694074 & -1.429547 \\ \mathrm{H} & -0.264809 & -0.763178 & 2.160309 \\ \mathrm{H} & -2.115497 & -1.703446 & 0.821246 \\ \mathrm{H} & -2.580146 & -0.068733 & 1.362468 \\ \mathrm{Li} & -0.274703 & 1.711694 & 0.812753 \\ \mathrm{H} & 2.806186 & -1.291384 & -0.514314\end{array}$




\section{c4CS03}

$\begin{array}{lrrr}1 & 1 & & \\ \mathrm{C} & -0.440312 & -0.891669 & -0.146649 \\ \mathrm{C} & 0.463037 & -0.800372 & 1.136895 \\ \mathrm{C} & 1.756245 & -0.140854 & 0.630320 \\ \mathrm{C} & 1.830921 & -0.626720 & -0.809817 \\ \mathrm{~N} & 0.429989 & -0.360942 & -1.235428 \\ \mathrm{C} & -1.726882 & -0.094068 & 0.009023 \\ \mathrm{H} & 0.673490 & -1.805207 & 1.511221 \\ \mathrm{H} & 2.640337 & -0.369223 & 1.228067 \\ \mathrm{O} & -2.776141 & -0.519311 & 0.398353 \\ \mathrm{O} & -1.552300 & 1.251800 & -0.302838 \\ \mathrm{H} & -0.746759 & -1.927294 & -0.320593 \\ \mathrm{H} & 0.000244 & -0.238835 & 1.952071 \\ \mathrm{O} & 1.582928 & 1.306880 & 0.534691 \\ \mathrm{H} & 1.828709 & 1.741246 & 1.362473 \\ \mathrm{H} & 0.208911 & -0.787086 & -2.132659 \\ \mathrm{H} & 2.096382 & -1.691258 & -0.843554 \\ \mathrm{H} & 2.539999 & -0.062223 & -1.419661 \\ \mathrm{Li} & 0.270840 & 1.654801 & -0.835577 \\ \mathrm{H} & -2.397706 & 1.709208 & -0.142922\end{array}$

\section{c4SB01}

$\begin{array}{lrrr}1 & 1 & & \\ \mathrm{C} & 0.174295 & 0.406252 & 0.934001 \\ \mathrm{C} & -0.791455 & -0.774290 & 1.143918 \\ \mathrm{C} & -1.754089 & -0.690778 & -0.055042 \\ \mathrm{C} & -1.976851 & 0.811162 & -0.216133 \\ \mathrm{~N} & -0.609928 & 1.403136 & 0.077915 \\ \mathrm{C} & 1.459905 & 0.084161 & 0.153074 \\ \mathrm{H} & -1.342259 & -0.657788 & 2.082640 \\ \mathrm{H} & -2.702263 & -1.207991 & 0.128398 \\ \mathrm{O} & 2.211657 & -0.830213 & 0.565112 \\ \mathrm{O} & 1.713053 & 0.799601 & -0.871953 \\ \mathrm{H} & 0.459537 & 0.886040 & 1.873013 \\ \mathrm{H} & -0.245248 & -1.718797 & 1.186261 \\ \mathrm{O} & -1.138698 & -1.126032 & -1.261282 \\ \mathrm{H} & -1.123074 & -2.092065 & -1.301383 \\ \mathrm{H} & -0.016002 & 1.505089 & -0.773607 \\ \mathrm{H} & -2.678710 & 1.195906 & 0.525596 \\ \mathrm{H} & -2.290537 & 1.095655 & -1.220511 \\ \mathrm{Li} & 3.308640 & -0.306141 & -0.924436 \\ \mathrm{H} & -0.676795 & 2.324534 & 0.513584\end{array}$




\section{c4SB02}

$\begin{array}{lrrr}1 & 1 & & \\ \mathrm{C} & 0.187204 & 0.430845 & 0.915354 \\ \mathrm{C} & -0.765389 & -0.758292 & 1.152235 \\ \mathrm{C} & -1.759737 & -0.693857 & -0.014901 \\ \mathrm{C} & -1.993015 & 0.815115 & -0.181951 \\ \mathrm{~N} & -0.609148 & 1.394081 & 0.032039 \\ \mathrm{C} & 1.475012 & 0.098138 & 0.143268 \\ \mathrm{H} & -1.292110 & -0.640501 & 2.103177 \\ \mathrm{H} & -2.694367 & -1.219329 & 0.206043 \\ \mathrm{O} & 2.267984 & -0.745579 & 0.620657 \\ \mathrm{O} & 1.679442 & 0.724425 & -0.949534 \\ \mathrm{H} & 0.463272 & 0.937807 & 1.842445 \\ \mathrm{H} & -0.220674 & -1.703134 & 1.171427 \\ \mathrm{O} & -1.107095 & -1.223116 & -1.161822 \\ \mathrm{H} & -1.751270 & -1.503869 & -1.825614 \\ \mathrm{H} & -0.040757 & 1.429278 & -0.843306 \\ \mathrm{H} & -2.651396 & 1.213116 & 0.593845 \\ \mathrm{H} & -2.359516 & 1.107522 & -1.166774 \\ \mathrm{Li} & 3.288181 & -0.372673 & -0.959197 \\ \mathrm{H} & -0.640795 & 2.341023 & 0.413637\end{array}$

c4SB03

$\begin{array}{lrrr}1 & 1 & & \\ \mathrm{C} & -0.206896 & 0.962650 & -0.421844 \\ \mathrm{C} & 0.705945 & 0.160716 & -1.381173 \\ \mathrm{C} & 1.713909 & -0.558223 & -0.461138 \\ \mathrm{C} & 2.007616 & 0.507617 & 0.603590 \\ \mathrm{~N} & 0.641546 & 1.137412 & 0.845585 \\ \mathrm{C} & -1.464216 & 0.182277 & -0.015299 \\ \mathrm{H} & 1.231256 & 0.846072 & -2.052930 \\ \mathrm{H} & 2.628665 & -0.820774 & -0.996534 \\ \mathrm{O} & -2.445188 & 0.150030 & -0.787508 \\ \mathrm{O} & -1.425789 & -0.442813 & 1.104206 \\ \mathrm{H} & -0.487848 & 1.941481 & -0.811956 \\ \mathrm{H} & 0.135185 & -0.539135 & -1.994149 \\ \mathrm{O} & 1.235885 & -1.765910 & 0.096730 \\ \mathrm{H} & 0.382821 & -1.644219 & 0.544013 \\ \mathrm{H} & 0.122085 & 0.612019 & 1.574938 \\ \mathrm{H} & 2.671877 & 1.288658 & 0.227673 \\ \mathrm{H} & 2.391890 & 0.101902 & 1.539830 \\ \mathrm{Li} & -3.244500 & -0.974113 & 0.552708 \\ \mathrm{H} & 0.709341 & 2.113765 & 1.139651\end{array}$


Section S3. Coordinates of the optimized structures of three most stable conformers of charge-solvated and salt-bridge of lithiated trans-4-hydroxyproline at B3LYP level with the basis set 6-31++G(d, p)

\section{t4SB01}

$\begin{array}{lrrr}1 & 1 & & \\ \mathrm{C} & -0.219325 & -0.281527 & -0.533548 \\ \mathrm{C} & 0.645156 & -1.135060 & 0.424921 \\ \mathrm{C} & 1.942991 & -0.320118 & 0.579456 \\ \mathrm{C} & 1.432648 & 1.115788 & 0.623874 \\ \mathrm{~N} & 0.405627 & 1.113301 & -0.490104 \\ \mathrm{C} & -1.690817 & -0.133918 & -0.133820 \\ \mathrm{H} & 0.823920 & -2.131209 & 0.016831 \\ \mathrm{O} & 2.748604 & -0.384684 & -0.593365 \\ \mathrm{O} & -2.402670 & -1.161556 & -0.043602 \\ \mathrm{O} & -2.125518 & 1.046783 & 0.085860 \\ \mathrm{H} & -0.164197 & -0.645096 & -1.561264 \\ \mathrm{H} & 0.149271 & -1.257057 & 1.392848 \\ \mathrm{H} & 2.507218 & -0.579282 & 1.481722 \\ \mathrm{H} & -0.384756 & 1.764556 & -0.322660 \\ \mathrm{H} & 2.198916 & 1.863241 & 0.418964 \\ \mathrm{H} & 0.915070 & 1.342177 & 1.557602 \\ \mathrm{Li} & -3.802060 & 0.086172 & 0.367451 \\ \mathrm{H} & 3.234368 & -1.219913 & -0.633561 \\ \mathrm{H} & 0.859726 & 1.335631 & -1.378552\end{array}$

\section{t4SB02}

$\begin{array}{lrrr}1 & 1 & & \\ \mathrm{C} & -0.218882 & -0.281416 & -0.529658 \\ \mathrm{C} & 0.647762 & -1.129445 & 0.430556 \\ \mathrm{C} & 1.944195 & -0.320502 & 0.574221 \\ \mathrm{C} & 1.431245 & 1.123768 & 0.623035 \\ \mathrm{~N} & 0.402647 & 1.116189 & -0.488336 \\ \mathrm{C} & -1.690943 & -0.134090 & -0.132963 \\ \mathrm{H} & 0.839199 & -2.123017 & 0.025436 \\ \mathrm{O} & 2.698646 & -0.504987 & -0.620755 \\ \mathrm{O} & -2.401664 & -1.161628 & -0.037310 \\ \mathrm{O} & -2.127795 & 1.047701 & 0.080121 \\ \mathrm{H} & -0.159143 & -0.648970 & -1.555900 \\ \mathrm{H} & 0.155434 & -1.242044 & 1.400486 \\ \mathrm{H} & 2.512309 & -0.588137 & 1.470160 \\ \mathrm{H} & -0.390552 & 1.764686 & -0.321669 \\ \mathrm{H} & 2.187052 & 1.882501 & 0.417594 \\ \mathrm{H} & 0.913534 & 1.352310 & 1.557484 \\ \mathrm{Li} & -3.802798 & 0.085249 & 0.363680 \\ \mathrm{H} & 3.645077 & -0.401028 & -0.454859 \\ \mathrm{H} & 0.853199 & 1.336056 & -1.379016\end{array}$




\section{t4SB03}

$\begin{array}{lrrr}1 & 1 & & \\ \mathrm{C} & 0.386281 & 0.060761 & 0.910462 \\ \mathrm{C} & -0.725088 & -0.967459 & 0.661046 \\ \mathrm{C} & -1.574873 & -0.356671 & -0.473454 \\ \mathrm{C} & -1.571198 & 1.146539 & -0.152739 \\ \mathrm{~N} & -0.217601 & 1.402328 & 0.475965 \\ \mathrm{C} & 1.652943 & -0.134348 & 0.058971 \\ \mathrm{H} & -1.357786 & -1.076331 & 1.547376 \\ \mathrm{O} & -2.922455 & -0.767762 & -0.475439 \\ \mathrm{O} & 2.309350 & -1.194115 & 0.176079 \\ \mathrm{O} & 1.983415 & 0.816062 & -0.729213 \\ \mathrm{H} & 0.682813 & 0.120923 & 1.959846 \\ \mathrm{H} & -0.301724 & -1.944028 & 0.417907 \\ \mathrm{H} & -1.104012 & -0.535931 & -1.449950 \\ \mathrm{H} & 0.478372 & 1.762772 & -0.211889 \\ \mathrm{H} & -2.347644 & 1.355092 & 0.586274 \\ \mathrm{H} & -1.700517 & 1.798549 & -1.016533 \\ \mathrm{Li} & 3.503860 & -0.366846 & -1.080394 \\ \mathrm{H} & -3.030728 & -1.591990 & -0.968404 \\ \mathrm{H} & -0.278014 & 2.068783 & 1.247668\end{array}$

\section{t4CS01}

$\begin{array}{lrrr}1 & 1 & & \\ \mathrm{C} & -0.291394 & -0.242791 & -0.491145 \\ \mathrm{C} & 0.577794 & -1.130864 & 0.451785 \\ \mathrm{C} & 1.892392 & -0.337677 & 0.549568 \\ \mathrm{C} & 1.387467 & 1.103795 & 0.573389 \\ \mathrm{~N} & 0.303661 & 1.136357 & -0.465348 \\ \mathrm{C} & -1.750748 & -0.180700 & -0.082065 \\ \mathrm{H} & 0.713445 & -2.135467 & 0.047437 \\ \mathrm{O} & 2.666671 & -0.459697 & -0.639792 \\ \mathrm{O} & -2.340230 & -1.360445 & -0.045935 \\ \mathrm{O} & -2.337941 & 0.871320 & 0.186452 \\ \mathrm{H} & -0.260549 & -0.639378 & -1.511208 \\ \mathrm{H} & 0.119926 & -1.227101 & 1.442098 \\ \mathrm{H} & 2.474541 & -0.593358 & 1.442096 \\ \mathrm{H} & 0.773998 & 1.270396 & -1.359360 \\ \mathrm{H} & 2.164827 & 1.829140 & 0.327527 \\ \mathrm{H} & 0.969595 & 1.345124 & 1.557001 \\ \mathrm{Li} & -1.255538 & 2.356877 & -0.090737 \\ \mathrm{H} & 3.161020 & -1.290338 & -0.640645 \\ \mathrm{H} & -3.276886 & -1.264157 & 0.209713\end{array}$




\section{t4CSO2}

$\begin{array}{lrrr}1 & 1 & & \\ \mathrm{C} & -0.290652 & -0.240139 & -0.489071 \\ \mathrm{C} & 0.579854 & -1.125768 & 0.453583 \\ \mathrm{C} & 1.891691 & -0.340352 & 0.547006 \\ \mathrm{C} & 1.387633 & 1.109842 & 0.573708 \\ \mathrm{~N} & 0.303421 & 1.140479 & -0.464351 \\ \mathrm{C} & -1.750495 & -0.179534 & -0.081509 \\ \mathrm{H} & 0.729603 & -2.127014 & 0.049148 \\ \mathrm{O} & 2.614696 & -0.586984 & -0.656368 \\ \mathrm{O} & -2.336103 & -1.360482 & -0.039785 \\ \mathrm{O} & -2.341239 & 0.872260 & 0.181280 \\ \mathrm{H} & -0.257919 & -0.638678 & -1.508483 \\ \mathrm{H} & 0.124368 & -1.217093 & 1.444352 \\ \mathrm{H} & 2.474281 & -0.607075 & 1.435336 \\ \mathrm{H} & 0.773219 & 1.270305 & -1.359186 \\ \mathrm{H} & 2.156342 & 1.846190 & 0.329139 \\ \mathrm{H} & 0.969332 & 1.353676 & 1.557744 \\ \mathrm{Li} & -1.261098 & 2.356934 & -0.094900 \\ \mathrm{H} & 3.556494 & -0.410720 & -0.530516 \\ \mathrm{H} & -3.273383 & -1.266393 & 0.214312\end{array}$

\section{t4CS03}

$\begin{array}{lrrr}1 & 1 & & \\ \mathrm{C} & 0.197641 & 0.151867 & -0.494737 \\ \mathrm{C} & -0.567734 & 1.059093 & 0.511491 \\ \mathrm{C} & -1.957702 & 0.413720 & 0.549078 \\ \mathrm{C} & -1.617634 & -1.083668 & 0.492946 \\ \mathrm{~N} & -0.469815 & -1.173949 & -0.453985 \\ \mathrm{C} & 1.655222 & -0.072134 & -0.117287 \\ \mathrm{H} & -0.607024 & 2.098087 & 0.183140 \\ \mathrm{O} & -2.632242 & 0.839842 & -0.632115 \\ \mathrm{O} & 2.501245 & 0.837263 & -0.079424 \\ \mathrm{O} & 1.954052 & -1.306172 & 0.173468 \\ \mathrm{H} & 0.171658 & 0.590305 & -1.497366 \\ \mathrm{H} & -0.111831 & 1.022042 & 1.506089 \\ \mathrm{H} & -2.516570 & 0.687491 & 1.451868 \\ \mathrm{H} & -0.798007 & -1.433757 & -1.379259 \\ \mathrm{H} & -2.445148 & -1.704615 & 0.143205 \\ \mathrm{H} & -1.312482 & -1.446865 & 1.480987 \\ \mathrm{Li} & 4.192676 & 1.263186 & 0.166109 \\ \mathrm{H} & -3.572111 & 0.620474 & -0.579077 \\ \mathrm{H} & 1.058985 & -1.785802 & 0.025606\end{array}$


Section S4. Coordinates of the optimized structure of the lowest energy lithiated cis-3-hydroxyproline structures for the charge solvated (C3CS01) and salt-bridge (C3SB01) conformations at M062X level with the basis set $6-31++\mathrm{G}(\mathrm{d}, \mathrm{p})$

\section{C3CS01}

$\begin{array}{lrrr}1 & 1 & & \\ \mathrm{C} & 0.135721 & -0.374555 & -0.603916 \\ \mathrm{C} & -0.660440 & 0.940468 & -0.501592 \\ \mathrm{C} & -2.098956 & 0.440600 & -0.619217 \\ \mathrm{C} & -2.066281 & -0.965014 & 0.027555 \\ \mathrm{~N} & -0.626971 & -1.195409 & 0.349042 \\ \mathrm{C} & 1.562852 & -0.208509 & -0.118204 \\ \mathrm{H} & -0.364346 & 1.686516 & -1.242164 \\ \mathrm{H} & -2.382996 & 0.383328 & -1.672919 \\ \mathrm{O} & 2.418176 & 0.054413 & -1.081260 \\ \mathrm{O} & 1.856903 & -0.275980 & 1.063771 \\ \mathrm{H} & 0.127153 & -0.736533 & -1.639407 \\ \mathrm{O} & -0.398274 & 1.422292 & 0.829384 \\ \mathrm{H} & -0.384176 & -2.181049 & 0.279875 \\ \mathrm{H} & -2.440152 & -1.721069 & -0.668234 \\ \mathrm{H} & -2.657158 & -1.025243 & 0.942980 \\ \mathrm{H} & 3.307668 & 0.196499 & -0.711753 \\ \mathrm{H} & -2.795256 & 1.121514 & -0.124967 \\ \mathrm{H} & -0.737060 & 2.317580 & 0.949630 \\ \mathrm{Li} & 0.154434 & -0.092475 & 1.946913\end{array}$

\section{C3SB01}

$\begin{array}{lrrr}1 & 1 & & \\ \mathrm{C} & -0.046342 & -0.224944 & -0.801478 \\ \mathrm{C} & -0.789084 & 1.080361 & -0.337553 \\ \mathrm{C} & -1.898430 & 0.575719 & 0.591661 \\ \mathrm{C} & -2.244485 & -0.808453 & 0.074064 \\ \mathrm{~N} & -0.888178 & -1.363099 & -0.286889 \\ \mathrm{C} & 1.333786 & -0.332398 & -0.161159 \\ \mathrm{H} & -1.208396 & 1.574253 & -1.221938 \\ \mathrm{H} & -2.762709 & 1.240278 & 0.605928 \\ \mathrm{O} & 2.235851 & 0.429616 & -0.567690 \\ \mathrm{O} & 1.479074 & -1.168322 & 0.777357 \\ \mathrm{H} & 0.037118 & -0.292751 & -1.887077 \\ \mathrm{O} & 0.024118 & 1.947902 & 0.393412 \\ \mathrm{H} & -0.943096 & -2.139284 & -0.950069 \\ \mathrm{H} & -2.836153 & -0.776230 & -0.843590 \\ \mathrm{H} & -2.724401 & -1.468966 & 0.796085 \\ \mathrm{H} & -1.487224 & 0.521814 & 1.605803 \\ \mathrm{H} & 0.714467 & 2.324263 & -0.170074 \\ \mathrm{Li} & 3.251345 & -0.382293 & 0.836763 \\ \mathrm{H} & -0.371406 & -1.710083 & 0.545025\end{array}$


Section S5. Coordinates of the optimized structure of the lowest energy lithiated cis-4-hydroxyproline structures for the charge solvated (C4CS01) and salt-bridge (C4SB01) conformations at M062X level with the basis set $6-31++\mathrm{G}(\mathrm{d}, \mathrm{p})$

\section{C4CS01}

$\begin{array}{lrrr}1 & 1 & & \\ \mathrm{C} & -0.410098 & 0.863172 & 0.260532 \\ \mathrm{C} & 0.420503 & 0.900416 & -1.062285 \\ \mathrm{C} & 1.697532 & 0.139846 & -0.690878 \\ \mathrm{C} & 1.890453 & 0.539057 & 0.760849 \\ \mathrm{~N} & 0.520142 & 0.300512 & 1.265352 \\ \mathrm{C} & -1.594521 & -0.071207 & 0.096373 \\ \mathrm{H} & 0.660594 & 1.933245 & -1.323349 \\ \mathrm{H} & 2.552930 & 0.359987 & -1.331867 \\ \mathrm{O} & -2.660550 & 0.522407 & -0.392783 \\ \mathrm{O} & -1.538940 & -1.267114 & 0.342523 \\ \mathrm{H} & -0.778197 & 1.860315 & 0.518819 \\ \mathrm{H} & -0.103471 & 0.452852 & -1.910520 \\ \mathrm{O} & 1.440301 & -1.279775 & -0.659111 \\ \mathrm{H} & 1.553111 & -1.674763 & -1.532383 \\ \mathrm{H} & 0.367489 & 0.699713 & 2.187401 \\ \mathrm{H} & 2.200795 & 1.588445 & 0.831416 \\ \mathrm{H} & 2.614297 & -0.090222 & 1.282665 \\ \mathrm{Li} & 0.239328 & -1.713919 & 0.812111 \\ \mathrm{H} & -3.376237 & -0.123240 & -0.528554\end{array}$

\section{C4SB01}

$\begin{array}{lrrr}1 & 1 & & \\ \mathrm{C} & -0.108158 & -0.404686 & 0.956450 \\ \mathrm{C} & 0.808854 & 0.808911 & 1.127055 \\ \mathrm{C} & 1.666012 & 0.758054 & -0.141535 \\ \mathrm{C} & 2.001999 & -0.721091 & -0.256713 \\ \mathrm{~N} & 0.689799 & -1.379518 & 0.104290 \\ \mathrm{C} & -1.400446 & -0.112652 & 0.182672 \\ \mathrm{H} & 1.432066 & 0.713192 & 2.020631 \\ \mathrm{H} & 2.570339 & 1.371599 & -0.086373 \\ \mathrm{O} & -2.106119 & 0.854481 & 0.533543 \\ \mathrm{O} & -1.701578 & -0.900107 & -0.762635 \\ \mathrm{H} & -0.375477 & -0.882299 & 1.901458 \\ \mathrm{H} & 0.215999 & 1.722482 & 1.209138 \\ \mathrm{O} & 0.885944 & 1.043640 & -1.285544 \\ \mathrm{H} & 0.716759 & 1.991371 & -1.354186 \\ \mathrm{H} & 0.087974 & -1.542441 & -0.727905 \\ \mathrm{H} & 2.751555 & -1.026558 & 0.474570 \\ \mathrm{H} & 2.296145 & -1.019458 & -1.262511 \\ \mathrm{Li} & -3.261503 & 0.218327 & -0.852831 \\ \mathrm{H} & 0.825006 & -2.281570 & 0.563153\end{array}$


Section S6. Coordinates of the optimized structure of the lowest energy lithiated trans-4-hydroxyproline structures for the charge solvated (T4CS01) and salt-bridge (T4SB01) conformations at M062X level with the basis set $6-31++\mathrm{G}(\mathrm{d}, \mathrm{p})$

\section{T4CS01}

$\begin{array}{lccc}1 & 1 & & \\ \mathrm{C} & -0.277539 & -0.237830 & -0.512427 \\ \mathrm{C} & 0.569648 & -1.152421 & 0.414812 \\ \mathrm{C} & 1.869494 & -0.354783 & 0.552565 \\ \mathrm{C} & 1.344202 & 1.072692 & 0.629838 \\ \mathrm{~N} & 0.319648 & 1.127127 & -0.454727 \\ \mathrm{C} & -1.727661 & -0.173238 & -0.088931 \\ \mathrm{H} & 0.717795 & -2.141889 & -0.019852 \\ \mathrm{O} & 2.623032 & -0.409856 & -0.643538 \\ \mathrm{O} & -2.306696 & -1.348269 & -0.024413 \\ \mathrm{O} & -2.309652 & 0.875822 & 0.170569 \\ \mathrm{H} & -0.257124 & -0.612914 & -1.540638 \\ \mathrm{H} & 0.092888 & -1.278230 & 1.392308 \\ \mathrm{H} & 2.459784 & -0.639329 & 1.429238 \\ \mathrm{H} & 0.840180 & 1.257148 & -1.321290 \\ \mathrm{H} & 2.119196 & 1.820214 & 0.455276 \\ \mathrm{H} & 0.871041 & 1.255025 & 1.600766 \\ \mathrm{Li} & -1.230770 & 2.356184 & -0.118276 \\ \mathrm{H} & 3.127006 & -1.230432 & -0.696081 \\ \mathrm{H} & -3.238334 & -1.256118 & 0.242100\end{array}$

\section{T4SB01}

$\begin{array}{lrrr}1 & 1 & & \\ \mathrm{C} & -0.207079 & -0.280365 & -0.570663 \\ \mathrm{C} & 0.631748 & -1.158859 & 0.381477 \\ \mathrm{C} & 1.904503 & -0.331840 & 0.598302 \\ \mathrm{C} & 1.361919 & 1.086281 & 0.673967 \\ \mathrm{~N} & 0.435760 & 1.091361 & -0.513294 \\ \mathrm{C} & -1.664625 & -0.122512 & -0.143209 \\ \mathrm{H} & 0.838290 & -2.135879 & -0.056991 \\ \mathrm{O} & 2.711516 & -0.329895 & -0.564437 \\ \mathrm{O} & -2.365881 & -1.147662 & -0.030160 \\ \mathrm{O} & -2.090224 & 1.051517 & 0.080468 \\ \mathrm{H} & -0.172064 & -0.635306 & -1.602083 \\ \mathrm{H} & 0.102917 & -1.318298 & 1.325528 \\ \mathrm{H} & 2.462307 & -0.611258 & 1.496731 \\ \mathrm{H} & -0.331272 & 1.778661 & -0.422244 \\ \mathrm{H} & 2.121784 & 1.858056 & 0.555806 \\ \mathrm{H} & 0.761228 & 1.257897 & 1.569180 \\ \mathrm{Li} & -3.744362 & 0.099332 & 0.401241 \\ \mathrm{H} & 3.216011 & -1.148708 & -0.642507 \\ \mathrm{H} & 0.981479 & 1.269397 & -1.360294\end{array}$


Section S7. Coordinates of the optimized structure of the lowest energy protonated cis-3-hydroxyproline structure at B3LYP level with the basis set 6-31++G(d, p)

$\begin{array}{lrrr}1 & 1 & & \\ \mathrm{C} & 0.165151 & -0.177912 & -0.701733 \\ \mathrm{C} & -0.675614 & 1.055075 & -0.302096 \\ \mathrm{C} & -2.114243 & 0.513662 & -0.375221 \\ \mathrm{C} & -2.004334 & -0.863088 & 0.283785 \\ \mathrm{~N} & -0.620815 & -1.360606 & -0.154843 \\ \mathrm{C} & 1.555941 & -0.241868 & -0.068918 \\ \mathrm{H} & -0.488971 & 1.883190 & -0.992477 \\ \mathrm{H} & -2.443658 & 0.432283 & -1.417244 \\ \mathrm{O} & 2.356699 & 0.693282 & -0.559170 \\ \mathrm{O} & 1.836743 & -1.077872 & 0.763236 \\ \mathrm{H} & 0.232687 & -0.265757 & -1.788304 \\ \mathrm{O} & -0.303994 & 1.372266 & 1.029244 \\ \mathrm{H} & -2.755236 & -1.588217 & -0.029207 \\ \mathrm{H} & -1.984863 & -0.794501 & 1.371211 \\ \mathrm{H} & -2.820210 & 1.157846 & 0.153864 \\ \mathrm{H} & -0.549032 & 2.281219 & 1.249898 \\ \mathrm{H} & -0.056263 & -1.736966 & 0.628919 \\ \mathrm{H} & -0.694178 & -2.106785 & -0.849933 \\ \mathrm{H} & 3.228446 & 0.645306 & -0.124214\end{array}$

Section S8. Coordinates of the optimized structure of the lowest energy protonated cis-4-hydroxyproline structure at B3LYP level with the basis set 6-31++G(d, p)

$\begin{array}{lrrr}1 & 1 & & \\ \mathrm{C} & -0.372157 & 0.286540 & -0.864022 \\ \mathrm{C} & 0.546201 & -0.951139 & -1.005513 \\ \mathrm{C} & 1.677072 & -0.695638 & 0.010031 \\ \mathrm{C} & 1.924012 & 0.803742 & -0.127654 \\ \mathrm{~N} & 0.511792 & 1.357022 & -0.229681 \\ \mathrm{C} & -1.573899 & 0.136816 & 0.075278 \\ \mathrm{H} & 0.946072 & -1.006737 & -2.022300 \\ \mathrm{H} & 2.582530 & -1.269815 & -0.212146 \\ \mathrm{O} & -2.399881 & -0.816928 & -0.338166 \\ \mathrm{O} & -1.721493 & 0.844588 & 1.048801 \\ \mathrm{H} & -0.733390 & 0.645196 & -1.830937 \\ \mathrm{H} & 0.004358 & -1.877161 & -0.805454 \\ \mathrm{O} & 1.228013 & -0.884526 & 1.344002 \\ \mathrm{H} & 1.276032 & -1.815655 & 1.600988 \\ \mathrm{H} & 0.477026 & 2.243001 & -0.738922 \\ \mathrm{H} & 2.452523 & 1.050981 & -1.049837 \\ \mathrm{H} & 2.423979 & 1.245497 & 0.734223 \\ \mathrm{H} & 0.096843 & 1.529802 & 0.707046 \\ \mathrm{H} & -3.168997 & -0.871250 & 0.259299\end{array}$


Table S1: Experimental and theoretical infrared peaks $\left(\mathrm{cm}^{-1}\right)$ along with the vibrational modes for each of the three lithiated hydroxyproline isomers

\begin{tabular}{|c|c|c|c|c|c|c|c|c|c|}
\hline Experimental & \multicolumn{2}{|c|}{ Theoretical } & Experimental & \multicolumn{2}{|c|}{ Theoretical } & Experimental & \multicolumn{2}{c|}{ Theoretical } & Tentative Band \\
Assignments \\
$\begin{array}{c}\text { Lithiated cis-3- } \\
\text { hydroxyproline }\end{array}$
\end{tabular}

